\begin{abstract}
H. S. Tzou
Department of Mechanical

Engineering

University of Kentucky

Lexington, KY 40506-0108

J. P. Zhong

United Technologies Carrier Co.

Syracuse, NY

\section{Spatially Filtered Vibration Control of Cylindrical Shells}

Distributed actuators offer spatially distributed actuations and they are usually effective to multiple modes of a continuum. Spatially filtered distributed vibration controls of a laminated cylindrical shell and a piezoelectric shell are investigated, and their control effectivenesses are evaluated in this study. In general, there are two control actions, the in-plane membrane control forces and the counteracting control moments, induced by the distributed actuator in the laminated shell. There is only an in-plane circumferential control force in the piezoelectric shell. Analyses suggest that in either case the control actions are effective in odd natural modes and ineffective in even modes. Spatially filtered control effectiveness and active damping of both shells are studied. (C) 1996 John Wiley \& Sons, Inc.
\end{abstract}

\section{INTRODUCTION}

Distributed sensors and actuators provide spatially distributed sensing and actuation to elastic continua-distributed systems. Unlike conventional discrete sensors and actuators, these distributed devices are usually responsive to spatially distributed phenomena; they are usually effective in sensing and controlling multiple natural modes of distributed systems (Tzou, 1993). There have been significant research and development activities on new piezoelectric devices in recent years (Baz and Poh, 1988; Hubbard and Burke, 1992; Lee and Moon, 1990; Birman, 1992; Tzou et al., 1993; Tzou and Fu, 1993). Distributed piezoelectric sensors and actuators are being increasingly used in active intelligent structures (Tzou and Anderson, 1992) and precision electromechanical systems (Tzou and Fukuda, 1992). However, detailed electromechanics and spatial filtering characteristics of distributed actuators are still not well understood. This article is intended to investigate the distributed vibration control behavior of spatially distributed piezoelectric actuators and their spatial filtering characteristics in vibration controls of cylindrical shells.

In general, distributed piezoelectric actuators either expand or contract in their sensitive directions when external voltages or charges are applied, the converse piezoelectric effect. Depending on the placement of the actuators on an elastic continuum, in-plane membrane control forces and/or counteracting control moments can be generated. In addition, because control voltage is also spatially distributed, this voltage can be surface averaged, resulting in a filtering effect to a few specific natural modes. This spatial filtering behavior is particularly significant for symmetrical geometries with symmetrical boundary conditions (Tzou, 1993). In this study, these spatially distributed control characteristics are discussed

Received January 23, 1995; Accepted October 13, 1995.

Shock and Vibration, Vol. 3, No. 4, p. 269-278 (1996)

(C) 1996 by John Wiley \& Sons, Inc.

CCC $1070-9622 / 96 / 040269-10$ 
and demonstrated in two cylindrical shells, a piezoelectric laminated cylindrical shell and a piezoelectric cylindrical shell. Mathematical modeling of these two cylindrical shells are presented first, followed by analyses of spatially filtered distributed control behavior of these two shells. Spatially filtered distributed controls of cylindrical shells are investigated in case studies.

\section{MODELING AND ANALYSIS}

Spatial filtering behaviors of two cylindrical shells were studied. The first cylindrical shell is a laminated composite in which an elastic cylindrical shell is sandwiched between two piezoelectric layers. One layer serves as a distributed sensor and the other as a distributed actuator that introduces control actions when external voltages are applied. The second cylindrical shell is made solely of a piezoelectric material and its control action is introduced via in-plane membrane forces. Detailed electromechanics and modeling are discussed in this section; spatially filtered control characteristics and effectiveness are discussed in the next section. Note that symmetrical hexagonal piezoelectric materials (Tzou, 1993; Tzou and Zhong, 1993) are used in both cases in this study.

\section{Laminated Cylindrical Shell Composite}

It is assumed that a spatially distributed piezoelectric actuator layer (with thickness $h^{\mathrm{a}}$ ) is laminated on the top surface of an elastic cylindrical shell (with thickness $h$; Table 1). The other dis-

Table 1. Dimensional and Material Properties of Laminated Cylindrical Shell

\begin{tabular}{cll}
\hline Properties & \multicolumn{1}{c}{ Steel } & \multicolumn{1}{c}{ PVDF } \\
\hline$L$ & $1.00 \times 10^{-1} \mathrm{~m}$ & $1.00 \times 10^{-2} \mathrm{~m}$ \\
$R$ & $5.00 \times 10^{-2} \mathrm{~m}$ & \\
$h$ & $1.00 \times{ }^{-3} \mathrm{~m}$ & $2.500 \times 10^{-5} \mathrm{~m}$ \\
$\beta$ & $60^{\circ}, 90^{\circ}, 120^{\circ}$ & \\
$\rho$ & $7.80 \times 10^{3} \mathrm{~kg} / \mathrm{m}^{3}$ & $1.800 \times 10^{3} \mathrm{~kg} / \mathrm{m}^{3}$ \\
$Y$ & $2.10 \times 10^{11} \mathrm{~N} / \mathrm{mm}^{2}$ & $1.600 \times 10^{9} \mathrm{~N} / \mathrm{mm}^{2}$ \\
$\mu$ & 0.300 & 0.290 \\
$d_{31}$ & & $6.000 \times 10^{-12} \mathrm{C} / \mathrm{N}$ \\
$d_{32}$ & & $6.000 \times 10^{-12} \mathrm{C} / \mathrm{N}$ \\
$d_{33}$ & & $13.000 \times 10^{-12} \mathrm{C} / \mathrm{N}$ \\
$\varepsilon / \varepsilon_{o}$ & & 10 \\
$\varepsilon_{o}$ & & $8.85 \times 10^{-10} \mathrm{~F} / \mathrm{m}$ \\
\hline
\end{tabular}

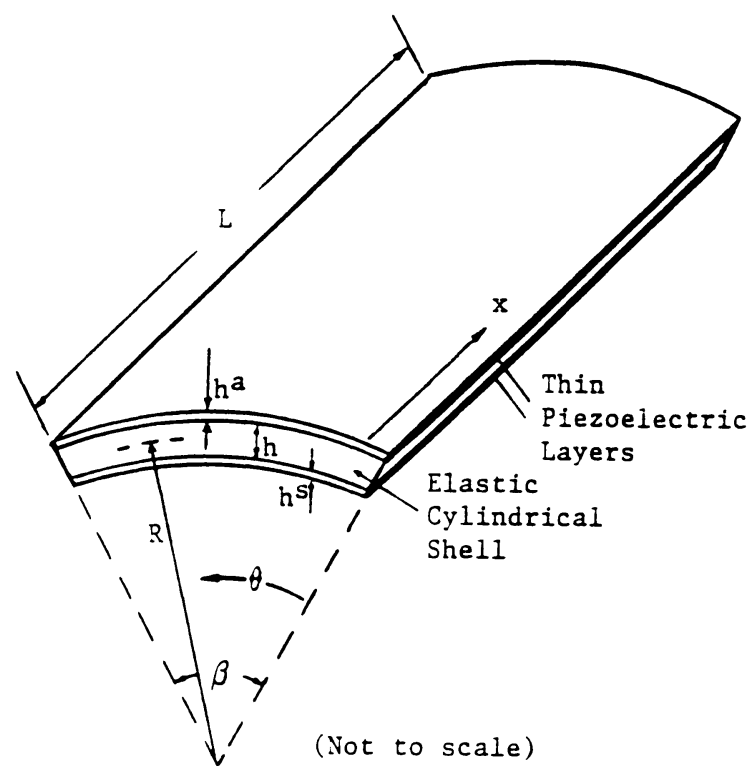

FIGURE 1 A cylindrical shell laminated with piezoelectric actuators.

tributed sensor layer is laminated on the bottom surface of the cylindrical shell. Active directions of the symmetrical hexagonal piezoelectric layers are aligned with the coordinate axes $x$ and $\theta$ of the cylindrical shell (Fig. 1). The cylindrical shell is assumed to be simply supported on all edges.

The laminated actuator layer introduces two control actions: in-plane membrane control forces and counteracting control moments. The effective axes of the actuator are aligned with the coordinate axes. One in-plane control force is in the $x$ direction and the other is in the $\theta$ direction, the circumferential direction. Furthermore, because the actuator layer is surface laminated, these in-plane control forces multiplied by the moment arms (distances from the neutral surface to the midplane of the actuator layers) yield counteracting control moments. A transverse electromechanical equation of the laminated cylindrical shell with distributed piezoelectric layers can be derived as (Zhong, 1991; Tzou, 1993)

$$
\begin{aligned}
& \frac{1}{R}\left\{-\frac{\partial}{\partial x}\left(\frac{\partial\left(M_{x x} R\right)}{\partial x}+\frac{\partial\left(M_{\theta x}\right)}{\partial \theta}+\frac{\partial\left(M_{x x}^{\mathrm{e}} R\right)}{\partial x}\right)\right. \\
& \left.-\frac{\partial}{\partial \theta}\left[\frac{1}{R}\left(\frac{\partial\left(M_{x \theta} R\right)}{\partial x}+\frac{\partial\left(M_{\theta \theta}\right)}{\partial \theta}+\frac{\partial\left(M_{\theta \theta}^{\mathrm{e}}\right)}{\partial \theta}\right)\right]\right\} \\
& \quad+\left[\frac{N_{\theta \theta}}{R}\right]+\rho h \ddot{u}_{3}=F_{3}-\left[\frac{N_{\theta \theta}^{\mathrm{e}}}{R}\right],
\end{aligned}
$$


where $R$ is the radius of the cylinder, $\rho$ is the mass density, $h$ is the shell thickness, and $F_{3}$ is the transverse excitation force. Resultant forces $N_{i j}$ and moments $M_{i j}$ can be defined by deformations on the neutral surface.

$$
\begin{aligned}
& N_{\theta \theta}=K\left\{\left(\frac{1}{R} \frac{\partial u_{\theta}}{\partial \theta}+\frac{u_{3}}{R}\right)+\mu\left(\frac{\partial u_{x}}{\partial x}\right)\right\} \\
& M_{x x}=D\left\{-\frac{\partial^{2} u_{3}}{\partial x^{2}}+\mu\left[\frac{1}{R} \frac{\partial}{\partial \theta}\left(\frac{u_{\theta}}{R}-\frac{1}{R} \frac{\partial u_{3}}{\partial \theta}\right)\right]\right\}, \\
& M_{\theta \theta}=D\left\{\left[\frac{1}{R} \frac{\partial}{\partial \theta}\left(\frac{u_{\theta}}{R}-\frac{1}{R} \frac{\partial u_{3}}{\partial \theta}\right)\right]-u\left(\frac{\partial^{2} u_{3}}{\partial x^{2}}\right)\right\},
\end{aligned}
$$

where $K$ is the (elastic) membrane stiffness, $K=$ $\left[Y h /\left(1-\mu^{2}\right)\right] ; D$ is the (elastic) bending stiffness, $D=\left[Y h^{3} / 12\left(1-\mu^{2}\right)\right] ; Y$ is Young's modulus; and $\mu$ is the Poisson ratio. $N_{\theta \theta}^{\mathrm{e}}, M_{x x}^{\mathrm{e}}$, and $M_{\theta \theta}^{\mathrm{e}}$ (with a superscript e) are the electrically induced control force and moments. Note that $N_{x x}^{\mathrm{e}}$ disappears from the equation because the curvature of the $x$ axis is zero or the radius is infinite. Controlled (forced) vibrations of cylindrical shells are derived using the modal expansion method. A velocity feedback control with spatially distributed control forces (the full state feedback) was proposed and its corresponding governing modal equations formulated.

Based on the modal expansion method, displacements in the three axial directions can be expressed in the modal expansion forms,

$$
\begin{aligned}
& u_{x}(x, \theta, t)=\sum_{m=1}^{\infty} \sum_{n=1}^{\infty} \eta_{m n} A_{m n} \cos \frac{m \pi x}{L} \sin \frac{n \pi \theta}{\beta}, \\
& u_{\theta}(x, \theta, t)=\sum_{m=1}^{\infty} \sum_{n=1}^{\infty} \eta_{m n} B_{m n} \sin \frac{m \pi x}{L} \cos \frac{n \pi \theta}{\beta}, \\
& u_{3}(x, \theta, t)=\sum_{m=1}^{\infty} \sum_{n=1}^{\infty} \eta_{m n} C_{m n} \sin \frac{m \pi x}{L} \sin \frac{n \pi \theta}{\beta},
\end{aligned}
$$

where $A_{m n}, B_{m n}$, and $C_{m n}$ are the relative oscillation amplitudes of the $m n$th mode; $\eta_{m n}$ is the modal participation factor; $m$ and $n$ are the halfwave numbers; $L$ is the shell length; and $\beta$ is the shell curvature angle. Note that there are three component natural frequencies for each $m n$ mode. The relative modal amplitude ratios $A_{m n}$, $B_{m n}$, and $C_{m n}$ of the $i$ th component natural frequency are defined as

$$
\frac{A_{i m n}}{C_{i m n}}=-\frac{k_{13}\left(\rho h \omega_{i m n}^{\prime 2}-k_{22}\right)-k_{12} k_{23}}{\left(\rho h \omega_{i m n}^{\prime 2}-k_{11}\right)\left(\rho h \omega_{i m n}^{\prime 2}-k_{22}\right)-k_{12}^{2}},
$$

$\frac{B_{i m n}}{C_{i m n}}=-\frac{k_{23}\left(\rho h \omega_{i m n}^{\prime 2}-k_{11}\right)-k_{21} k_{13}}{\left(\rho h \omega_{i m n}^{\prime 2}-k_{11}\right)\left(\rho h \omega_{i m n}^{\prime 2}-k_{22}\right)-k_{12}^{2}}$,

where $\omega_{i m n}^{\prime}$ is the $i$ th component natural frequency, and $k_{i j}$ are constants defined by the membrane stiffness, bending stiffness, Young's modulus, Poisson's ratio, radius, length, and half-wave numbers $m$ and $n$ (Tzou, 1993). Because the transverse oscillation usually is more important than the other two in-plane oscillations, only the transverse vibration

$$
u_{3}(x, \theta, t)=\sum_{m=1} \sum_{n=1} \eta_{m n}(t) C_{m n} \sin \frac{m \pi x}{L} \sin \frac{n \pi \theta}{\beta}
$$

is considered in the later analysis.

Using the modal expansion method and considering a viscous damping, one can derive the transverse modal equation for the simply supported laminated cylindrical shell with distributed actuator filters as

$$
\begin{aligned}
\ddot{\eta}_{m n}+\frac{c_{m n}}{\rho h} \dot{\eta}_{m n}+\omega_{m n}^{2} \eta_{m n} & =\frac{1}{\rho h N_{m n}} \int_{x} \int_{\theta}\left(\frac{N_{\theta \theta}^{\mathrm{e}}}{R}-\frac{\partial^{2} M_{x x}^{\mathrm{e}}}{\partial x^{2}}-\frac{1}{R^{2}} \frac{\partial^{2} M_{\theta \theta}^{\mathrm{e}}}{\partial \theta^{2}}\right) \\
& \sin \frac{m \pi x}{L} \sin \frac{n \pi \theta}{\beta} R d x d \theta
\end{aligned}
$$

where $c_{m n}$ is the damping constant and $N_{m n}$ for a simply supported cylindrical shell is defined by

$$
N_{m n}=\int_{0}^{L} \int_{0}^{\beta}\left(\sin \frac{m \pi x}{L} \sin \frac{n \pi \theta}{\beta}\right)^{2} R d x d \theta=\frac{L R \beta}{4} .
$$

It can be observed that all electric force/moment components are lumped on the right side of the modal equation and these force/moment components can be used to control the shell dynamics. Recall that the actuator layer is laminated on the surface of the cylindrical shell. Accordingly, the electric membrane force $N_{\theta \theta}^{\mathrm{e}}$ and bending moments $\left(M_{x x}^{\mathrm{e}}\right.$ and $\left.M_{\theta \theta}^{\mathrm{e}}\right)$ can be calculated when control voltages are known (Tzou, 1991). Distributed filtering characteristics of actuator actions, i.e., control forces and moments, are evaluated in the next section.

\section{Piezoelectric Cylindrical Shell}

In this case, the cylindrical shell is made entirely of a piezoelectric material. The inherent electric 
components in the system equation are intended to counteract the mechanical components such that the shell is actively controlled. The inherent electric components, e.g., membrane forces and moments, that are externally activated can be used for structural actuation and control. In the electromechanical equation of transverse oscillation, the electric membrane forces are divided by radii such that these terms vanish when the radii are infinite, e.g., plates, etc. Thus, structural control with the electric membrane forces is only effective for shell-type structures with nonzero curvatures. Again, it is assumed that the piezoelectric cylindrical shell is simply supported on all four edges (Fig. 2). Damping control of the piezoelectric cylindrical shell via distributed inplane membrane forces was investigated.

It is assumed that the thin piezoelectric cylindrical shell is made of a symmetrical hexagonal piezoelectric material, i.e., class $C_{6 v}=6 \mathrm{~mm}$, which has 10 material constants (Tzou, 1993; Tzou and Zhong, 1993). The top and bottom surfaces are covered by thin electrodes with negligible material properties. A transverse electric field $E_{3}$ is applied across the electroded surfaces. The transverse piezoelastic equation of the piezoelectric cylindrical shell is

$$
\begin{gathered}
D^{\prime}\left(-\frac{\partial^{4} u_{3}}{\partial x^{4}}+\frac{1}{R^{2}} \frac{\partial^{3} u_{3}}{\partial x^{2} \partial \theta}-\frac{2}{R^{2}} \frac{\partial^{4} u_{3}}{\partial x^{2} \partial \theta^{2}}+\frac{1}{R^{4}} \frac{\partial^{3} u_{3}}{\partial \theta^{3}}-\frac{1}{R^{4}} \frac{\partial^{4} u_{3}}{\partial \theta^{4}}\right) \\
-K^{\prime}\left(\frac{\mu}{R} \frac{\partial u_{x}}{\partial x}+\frac{1}{R^{2}} \frac{\partial u_{\theta}}{\partial \theta}+\frac{u_{3}}{R^{2}}\right)-\rho h \ddot{u}_{3}=\frac{N_{\theta \theta}^{\mathrm{e}}}{R}
\end{gathered}
$$

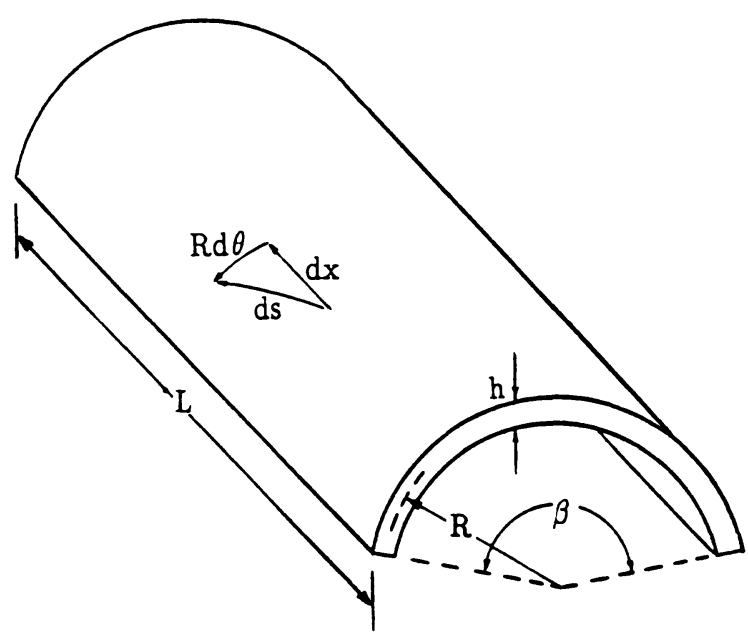

FIGURE 2 A piezoelectric cylindrical shell. where $D^{\prime}$ is the piezoelastic bending stiffness and $K^{\prime}$ the piezoelastic membrane stiffness. They are defined as

$$
\begin{aligned}
& D^{\prime}=D+\frac{e_{31}^{2} I}{\varepsilon_{33}}, \\
& K^{\prime}=K+\frac{e_{31}^{2} h}{\varepsilon_{33}},
\end{aligned}
$$

where $e_{31}$ is the piezoelectric constant, $\varepsilon_{33}$ is the dielectric constant, and $I$ is the area moment of inertia. As defined previously, $K$ is the elastic membrane stiffness, $K=\left[Y h /\left(1-\mu^{2}\right)\right] ; D$ is the elastic bending stiffness, $D=\left[Y h^{3} / 12\left(1-\mu^{2}\right)\right]$.

Note that the terms inside the first parentheses of the piezoelastic cylindrical shell equation are contributed by the bending effects and those inside the second parentheses are related to the inplane membrane effects. Because the radius of curvature $R$ of the $\theta$ axis (the circumferential direction) is not infinite, an in-plane electric membrane force $N_{\theta \theta}^{\mathrm{e}}$ is left in the piezoelastic equation and this force can be used to control shell dynamics. In addition, there is no counteracting control moment, because the membrane control force is on its neutral surface. The externally induced control force can be defined as

$$
N_{\theta \theta}^{\mathrm{e}}=\int_{\alpha_{3}} e_{31} E_{3} d \alpha_{3},
$$

where the electric field $E_{3}$ can be determined by either an open-loop or a closed-loop control. The electric field $E_{3}$ can be made as a function of either displacement or velocity in a closed-loop control system. In this study, a velocity feedback control was considered and its effect on damping control evaluated. Note that the feedback voltage is normalized with respect to the modal oscillation amplitudes in the later analysis.

Similar to the first case, the natural modes $U_{x}(x, \theta), U_{\theta}(x, \theta)$, and $U_{3}(x, \theta)$ of the simply supported piezoelectric cylindrical shell in three axial directions are

$$
\begin{aligned}
& U_{x}(x, \theta)=A_{m n} \cos \frac{m \pi x}{L} \sin \frac{n \pi \theta}{\beta}, \\
& U_{\theta}(x, \theta)=B_{m n} \sin \frac{m \pi x}{L} \cos \frac{n \pi \theta}{\beta}, \\
& U_{3}(x, \theta)=C_{m n} \sin \frac{m \pi x}{L} \sin \frac{n \pi \theta}{\beta},
\end{aligned}
$$


where $A_{m n}, B_{m n}$, and $C_{m n}$ are constants, i.e., amplitudes of modal oscillations; and $m$ and $n$ are integers, the half-wave numbers.

\section{SPATIALLY FILTERED DISTRIBUTED CONTROL}

As discussed previously, electrically controlled membrane control forces and bending moments are used to influence the shell dynamics. In the laminated shell case, there are two control actions: membrane control forces and control moments. In the piezoelectric shell case, the control action comes from an in-plane electric circumferential force. A generic closed-loop control schematic of piezoelectric systems is illustrated in Figure 3 . In this section, spatially distributed control of these two cylindrical shells is discussed.

\section{Laminated Cylindrical Shell Composite}

As defined previously, a fully distributed piezoelectric actuator layer covers the whole top surface of the cylindrical shell and a feedback voltage $\phi_{3}^{\mathrm{a}}$ is injected into the distributed actuator. Using the modal expansion technique and the equivalent control moment approach (Tzou and $\mathrm{Fu}, 1993$ ), one can derive the control forces and moments in the modal domain.

$$
\begin{aligned}
& \int_{0}^{L} \int_{0}^{\beta} N_{\theta \theta}^{\mathrm{e}} \sin \frac{m \pi x}{L} \sin \frac{n \pi \theta}{\beta} d x d \theta \\
& =\frac{4 e_{31} L \beta}{\pi^{2}} \frac{1}{m n} \phi_{3}^{\mathrm{a}} \hat{\delta}_{m n}, \\
& \int_{0}^{L} \int_{0}^{\beta} \frac{\partial^{2} M_{x x}^{\mathrm{e}}}{\partial x^{2}} \sin \frac{m \pi x}{L} \sin \frac{n \pi \theta}{\beta} R d x d \theta \\
& =-\frac{e_{31}\left(h+h^{\mathrm{a}}\right)}{2} \frac{4 m \beta R}{n L} \phi_{3}^{\mathrm{a}} \hat{\delta}_{m n},
\end{aligned}
$$

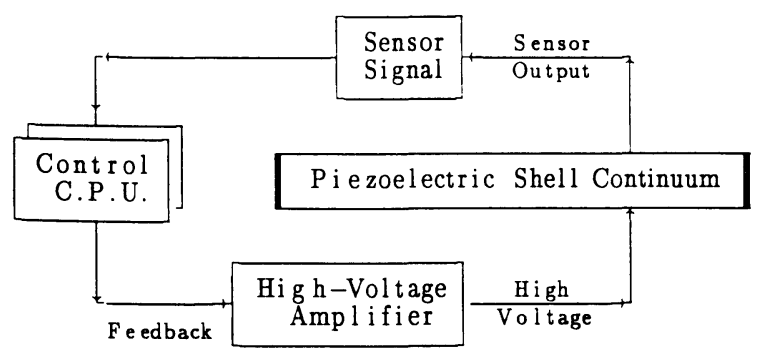

FIGURE 3 A closed-loop control schematic of piezoelectric systems.

$$
\begin{aligned}
\int_{0}^{L} \int_{0}^{\beta} \frac{1}{R} \frac{\partial^{2} M_{\theta \theta}^{\mathrm{e}}}{\partial \theta^{2}} & \sin \frac{m \pi x}{L} \sin \frac{n \pi \theta}{\beta} d x d \theta \\
& =-\frac{e_{31}\left(h+h^{\mathrm{a}}\right)}{2} \frac{4 n L}{m \beta R} \phi_{3}^{\mathrm{a}} \hat{\delta}_{m n},
\end{aligned}
$$

where

$$
\begin{aligned}
\hat{\delta}_{m n} & =\frac{1}{4}[1-\cos (m \pi)][1-\cos (n \pi)] \\
& = \begin{cases}1, & \text { if both } m \text { and } n \text { are odd integers, } \\
0, & \text { for other } m \text { and } n .\end{cases}
\end{aligned}
$$

Note that the resultant control force and moments are zero for all even modes. Thus, this fully distributed actuator is only effective on odd natural modes; control actions for even modes are filtered out due to voltage cancellations of antisymmetrical modes. Substituting the resultant forces and moments into the modal equation and imposing the full state velocity feedback, i.e., $\phi_{3}^{\mathrm{a}}=-\mathscr{G}_{\mathrm{fp}}^{\mathrm{vf}} \cdot \dot{\eta}_{m n}(t)$, one can derive the closedloop modal equation,

$$
\begin{aligned}
& \ddot{\eta}_{m n}+\frac{1}{\rho h} \\
& \left\{c_{m n}+\frac{16 e_{31}}{L R \beta}\left(\frac{L \beta}{\pi^{2}} \frac{1}{m n}+\frac{h+h^{\mathrm{a}}}{2}\left(\frac{m \beta R}{n L}+\frac{n L}{m \beta R}\right)\right) \mathscr{G}_{\mathrm{fp}}^{\mathrm{vf}} \hat{\delta}_{m n}\right\} \\
& \dot{\eta}_{m n}+\omega_{m n}^{2} \eta_{m n}=0,
\end{aligned}
$$

where $\mathscr{G}_{\mathrm{fp}}^{\mathrm{vf}}$ is the system feedback gain including the sensor sensitivity $S_{\mathrm{fp}}$, the amplifier gain $\mathscr{G}_{\mathrm{am}}$, and the gain of a differentiator circuit $\mathscr{G}_{\mathrm{de}}$, i.e., $\mathscr{G}_{\mathrm{fp}}^{\mathrm{vf}}=S_{\mathrm{fp}} \mathscr{G}_{\mathrm{am}} \mathscr{G}_{\mathrm{de}}$. An equivalent controlled modal damping ratio $\zeta_{m n}^{\prime}$ is written as

$$
\begin{aligned}
& \zeta_{m n}^{\prime}=\zeta_{m n}+\frac{8 e_{31}}{L R \beta \rho h \omega_{m n}} \\
& \left\{\frac{L \beta}{\pi^{2}} \frac{1}{m n}+\frac{h+h^{\mathrm{a}}}{2}\left(\frac{m \beta R}{n L}+\frac{n L}{m \beta R}\right)\right\} \mathscr{G}_{\mathrm{fp}}^{\mathrm{vf} \hat{\delta}_{m n},}
\end{aligned}
$$

and the initial damping ratio $\zeta_{m n}=c_{m n} / 2 \rho h \omega_{m n}$. The first term in the braces is associated with the membrane control action and the other two are associated with the bending control action. Apparently, the velocity feedback can be used to adjust the modal damping ratio. It should be pointed out that the sensor signal is a modal signal and it is directly contributed by strains and indirectly contributed by displacements or deformations (Tzou, 1991, 1993; Zhong, 1991). 


\section{Piezoelectric Cylindrical Shell}

Using the modal expansion technique and considering the velocity feedback (Tzou and Fu, 1993), one can derive the $m n$th modal equation as

$\ddot{\eta}_{m n}+\frac{1}{\rho h}\left(c_{m n}+\frac{16 e_{31} \phi_{3}}{3 R \pi^{2}} \delta^{\prime}(m, n)\right) \dot{\eta}_{m n}+\omega_{m n}^{2} \eta_{m n}=0$.

Again, $\eta_{m n}$ is the modal coordinate (the modal participation factor); $c_{m n}$ is the inherent viscous damping factor; $\phi_{3}$ is the resultant feedback voltage; and

$\delta^{\prime}(m, n)=(-\cos m \pi+1) \cdot(-\cos n \pi+1)$.

Thus, the equivalent controlled damping ratio $\zeta_{m n}^{\prime}$ of the piezoelectric shell with the velocity feedback becomes

$$
\begin{aligned}
\zeta_{m n}^{\prime} & =\frac{1}{2 \rho h \omega_{m n}}\left(c_{m n}+\frac{16 e_{31} \phi_{3}}{3 R \pi^{2}} \delta^{\prime}(m, n)\right) \\
& =\zeta_{m n}+\frac{1}{2 \rho h \omega_{m n}}\left(\frac{16 e_{31} \phi_{3}}{3 R \pi^{2}}\right) \delta^{\prime}(m, n) .
\end{aligned}
$$

The initial $m n$th modal damping ratio $\zeta_{m n}$ is defined as $\left(c_{m n} /\left(2 \rho h \omega_{m n}\right)\right)$. It is observed that the enhanced damping, the second term $\left(16 e_{31} \phi_{3} /\right.$ $\left.3 R \pi^{2}\right) \delta^{\prime}(m, n)$, vanishes for all even $m$ or $n$ modes because $\delta^{\prime}(m, n)=0$. Thus, this distributed inplane circumferential control force is only effective to odd natural modes (both $m$ and $n$ are odd numbers) of the piezoelectric cylindrical shell. Substituting all material and geometry parameters into the above equation, one can estimate the damping changes influenced by the resultant feedback voltage $\phi_{3}$.

\section{RESULTS AND DISCUSSION}

Theoretical analyses of the laminated cylindrical shell composite and the piezoelectric cylindrical shell presented above suggest that the fully distributed actuator layer only controls the odd natural modes of the laminated cylindrical shell, and the in-plane circumferential control force also only controls the odd natural modes of the piezoelectric shell. All even modes are filtered out from the control action, due to control signal cancellations and modal symmetries. Detailed control ef-
Table 2. Dimensional and Material Properties

\begin{tabular}{lll}
\hline Properties & \multicolumn{1}{c}{ Steel } & \multicolumn{1}{c}{ PVDF } \\
\hline$\rho\left(\mathrm{kg} / \mathrm{m}^{3}\right)$ & $7.80 \times 10^{3}$ & $1.80 \times 10^{3}$ \\
$Y\left(\mathrm{~N} / \mathrm{mm}^{2}\right)$ & $2.10 \times 10^{11}$ & $1.600 \times 10^{9}$ \\
$L(\mathrm{~m})$ & $1.00 \times 10^{-1}$ & $1.00 \times 10^{-2}$ \\
$R(\mathrm{~m})$ & $5.00 \times 10^{-2}$ & \\
$h(\mathrm{~m})$ & $1.00 \times 10^{-3}$ & $2.50 \times 10^{-5}$ \\
$\beta\left({ }^{\circ}\right)$ & $60,90,120$ & \\
$\mu$ & 0.30 & 0.290 \\
$d_{31}, d_{32}(\mathrm{C} / \mathrm{N})$ & & $6.00 \times 10^{-12}$ \\
$d_{33}(\mathrm{C} / \mathrm{N})$ & & $13.00 \times 10^{-12}$ \\
$\varepsilon_{o}(\mathrm{~F} / \mathrm{m})$ & & $8.85 \times 10^{-10}$ \\
$\varepsilon / \varepsilon_{o}$ & & 10 \\
\hline
\end{tabular}

fectiveness, via numerical simulations, of these two cylindrical shells is presented in this section.

\section{Laminated Cylindrical Shell Composite}

The elastic cylindrical shell was made of steel with a thickness of $1 \mathrm{~mm}$. The piezoelectric actuator layer was made of a $25-\mu \mathrm{m}$ polymeric polyvinylidene fluoride (PVDF) material and its mechanical properties were neglected in the analysis. Detailed material properties are listed in Table 2 . Note that there were three curvature angles $\left(60^{\circ}, 90^{\circ}\right.$, and $\left.120^{\circ}\right)$ considered in this analysis from which the effect of shell deepness could be evaluated. Filtered vibration (damping) controls, via the fully distributed actuator, of the laminated cylindrical shell with $60^{\circ}, 90^{\circ}$, and $120^{\circ}$ curvature angles are presented in this section. Total control effects resulting from the electric control membrane force and bending moments of the first three odd modes, $(1,1),(1,3)$, and $(3,1)$, are plotted in Fig. 4-6, and their filtering characteristics are

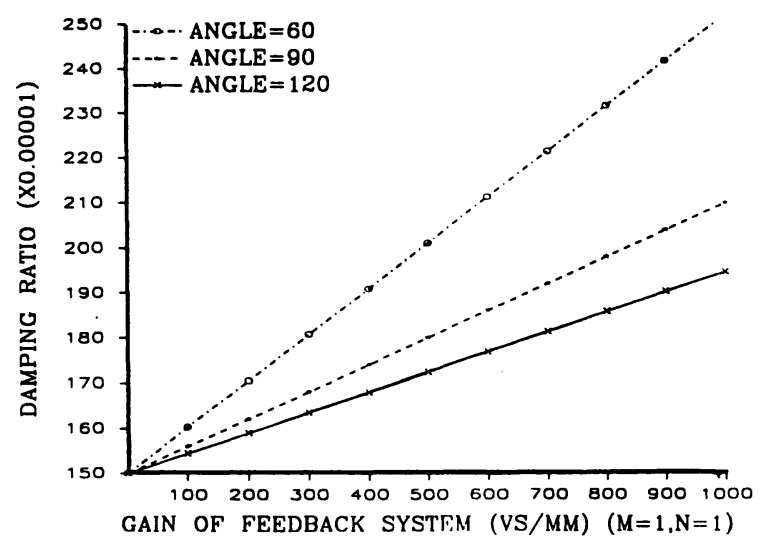

FIGURE 4 Damping control of the laminated shell, $(1,1)$ mode; (1-mm shell, $25-\mu \mathrm{m}$ actuator). 


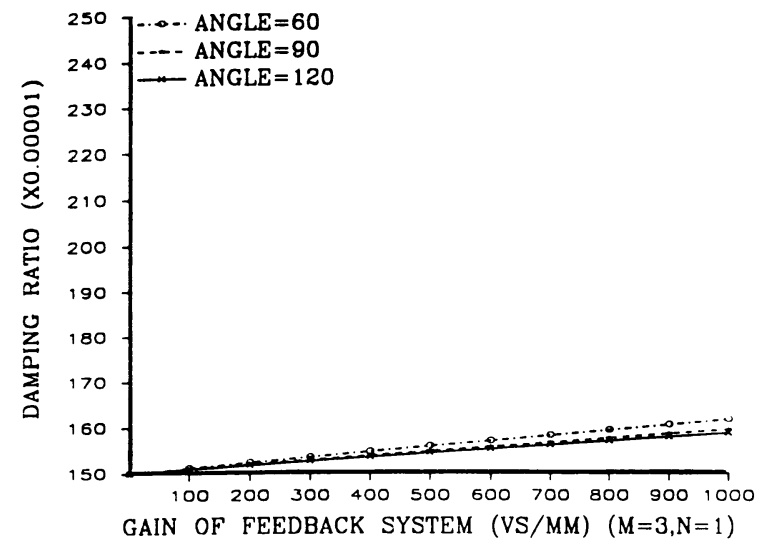

FIGURE 5 Damping control of the laminated shell, $(3,1)$ mode.

compared. (Comparisons of individual membrane and bending control effects are presented later.) Note that the fully distributed actuator $(25 \mu \mathrm{m})$ is only effective to odd modes and ineffective to even modes. Control effects due to thickness variations of the PVDF actuator layer and the cylindrical shell were also evaluated.

In general, the controlled damping ratio increases (linearly) as the system feedback gain increases, although the increase is relatively small. Note that the initial damping ratio was assumed to be $1 \%$ for all natural modes. Control effect from the electric membrane forces was larger than that from the electric bending moments, but they were in the same order. Figure $7(a, b)$ shows the membrane and bending control effect to the first mode (Fig. 4 illustrates the total control effects.) It was observed that as the mode increases, the membrane force contribution decreases more significantly than the moment contribution.

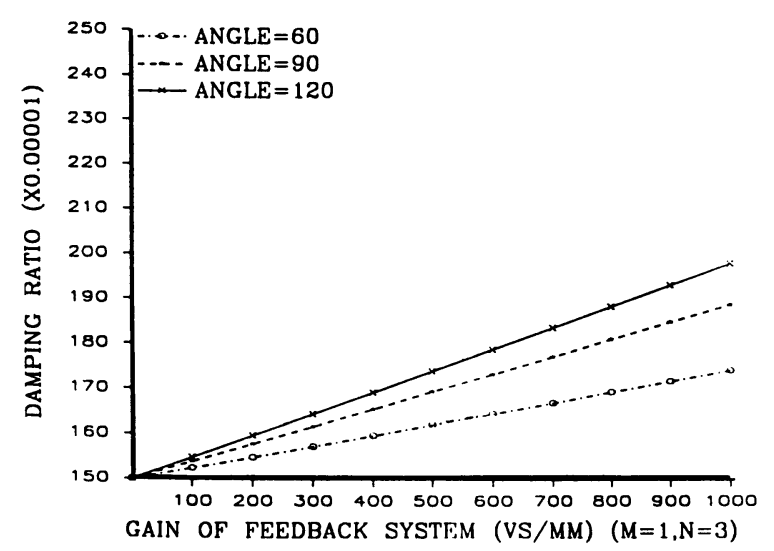

FIGURE 6 Damping control of the laminated shell, $(1,3)$ mode.
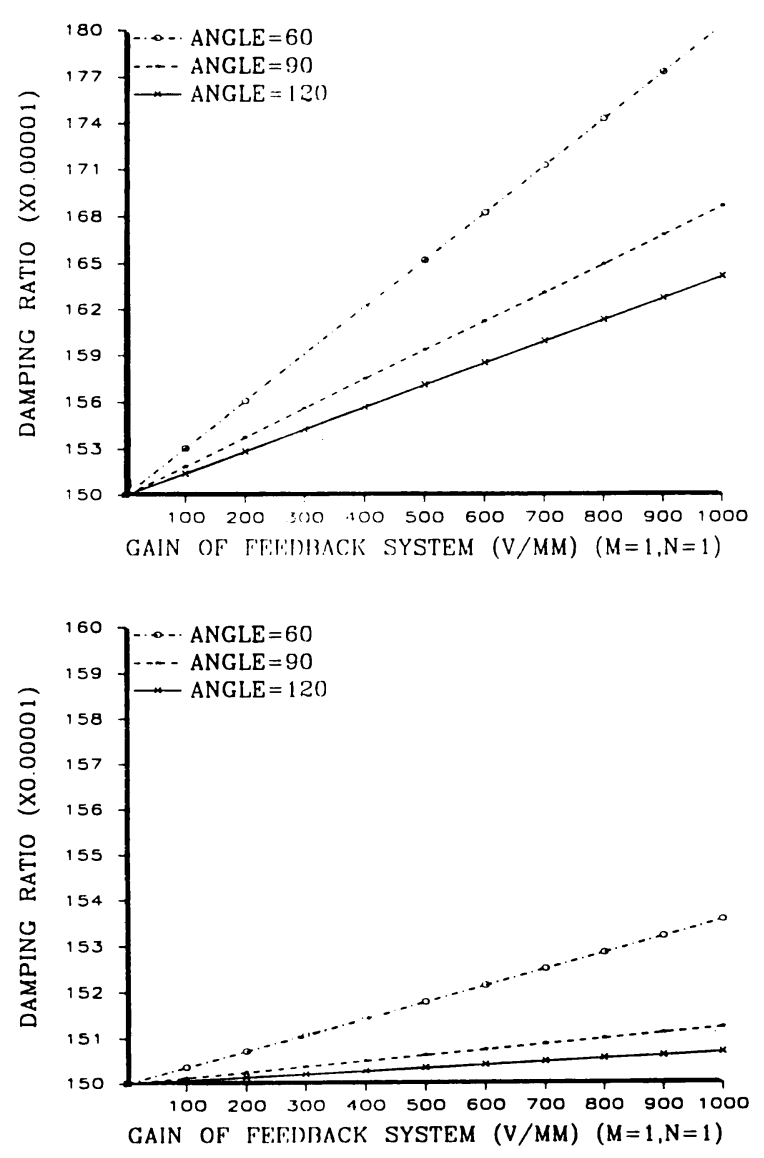

FIGURE 7 (a) Membrane control effects, $(1,1)$ mode. (b) Bending control effects, $(1,1)$ mode.

Note that the resultant convergence of a modal time history was determined by a product of the modal damping ratio and the natural frequency, i.e., $e^{-\zeta_{m n} \omega_{m n} t}$. Also, because there was a natural frequency in the denominator of the equivalent controlled damping ratio, shells with higher natural frequencies showed lower control effects, e.g., the $120^{\circ}$ shell in the $(1,1)$ and $(3,1)$ modes (Fig. 4,5$)$ and the $60^{\circ}$ shell in the $(1,3)$ mode (Fig. 6). Other simulation results showed that a thicker piezoelectric actuator contributes a better control effect. Figure 8 illustrates the damping controls of the laminated cylindrical shell with a 40- $\mu \mathrm{m}$ piezoelectric actuator layer. Comparing with Fig. 4, one can observe that the overall damping control was slightly improved.

In addition, a thinner elastic shell $(0.5 \mathrm{~mm})$ with $25-\mu \mathrm{m}$ piezoelectric actuators was also investigated (Fig. 9). Simulation results suggest that the membrane control action remained identical and the moment control action increased linearly when the elastic shell became thicker. However, 


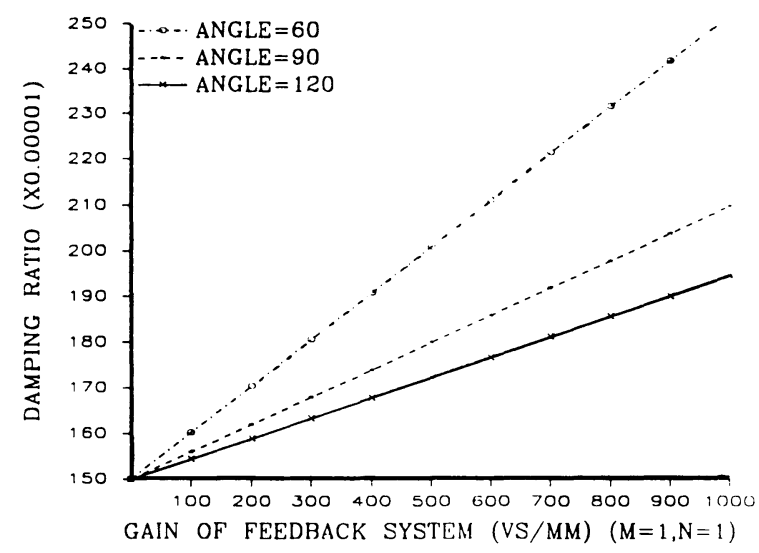

FIGURE 8 Damping control of the $(1,1)$ mode (1-mm shell, 40- $\mu \mathrm{m}$ actuator).

the overall control effect decreased because the elastic flexural rigidity was a cubic function of the shell thickness and the moment was only a linear function (Zhong, 1991; Tzou and Fu, 1993; Tzou, 1993).

\section{Piezoelectric Cylindrical Shell}

The piezoelectric cylindrical shell was made of a polymeric piezoelectric PVDF material with $e_{31}=e_{32}=4.6 \times 10^{-2} \mathrm{C} / \mathrm{m}^{2}, \varepsilon_{33}=1.026 \times 10^{-6}$ $\mathrm{F} / \mathrm{m}$, and a capacitance $C=3.8 \times 10^{-6} \mathrm{~F} / \mathrm{m}$. The shell dimension was $10 \mathrm{~cm}$ long, $1 \mathrm{~mm}$ thick, and the radius of curvature was $5 \mathrm{~cm}$. Three different curvature angles $\left(60^{\circ}, 90^{\circ}\right.$, and $\left.120^{\circ}\right)$ were studied and effects of shell deepness compared. Note that because the shell was made of the PVDF polymeric material, $D^{\prime} \cong D$ and $K^{\prime} \cong K$.

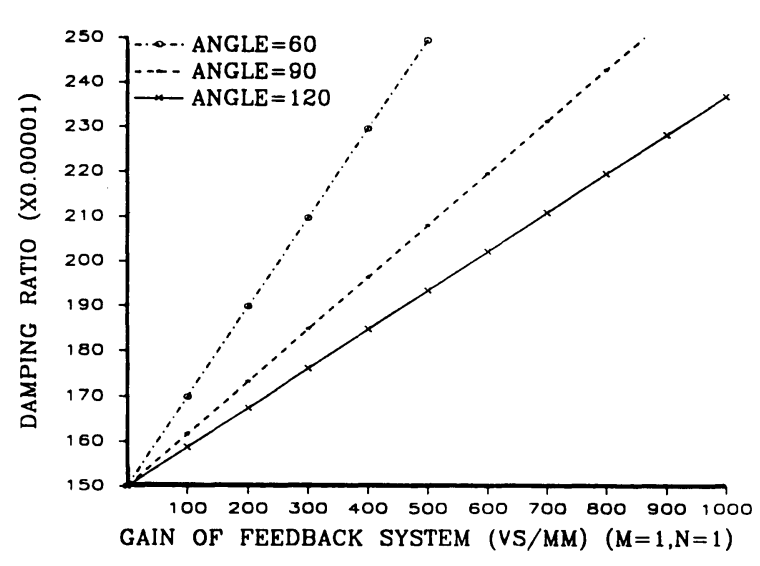

FIGURE 9 Damping control of the $(1,1)$ mode (0.5-mm shell, 25- $\mu \mathrm{m}$ actuator).

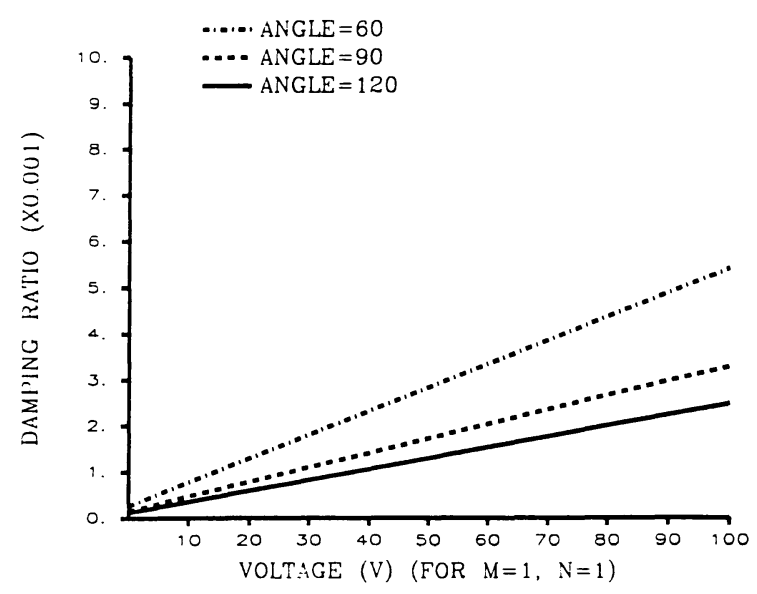

FIGURE 10 Damping control of the piezoelectric shell, $(1,1)$ mode.

Recall that $m$ denotes the half-wave number in the $x$ direction and $n$ the $\theta$ direction. The transverse natural mode is

$$
U_{3}(x, \theta)=C \sin \frac{m \pi x}{L} \sin \frac{n \pi \theta}{\beta} .
$$

Damping variations of the first three odd modes for all three curvature angles are plotted in Figs. 10-12. Damping ratios of the first three odd modes are controlled by the feedback voltages and their control effect increases as the feedback voltage increases. However, in practice, this voltage is limited by a breakdown voltage of the piezoelectric material. Figure 11 shows the control effect to the $(m=3, n=1)$ mode, i.e., sin $(3 \pi x / L) \sin (\pi \theta / \beta)$. Because the control forces were circumferential, i.e., in the $\theta$ direction, the

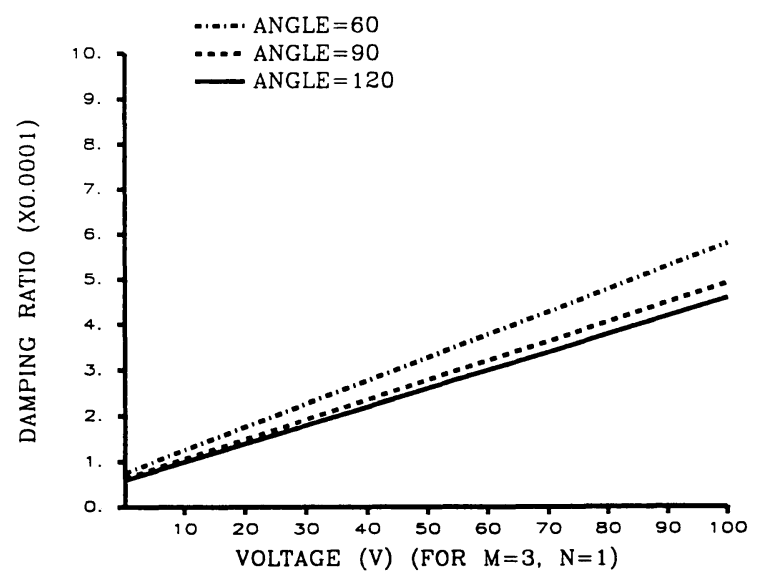

FIGURE 11 Damping control of the piezoelectric shell, $(3,1)$ mode. 


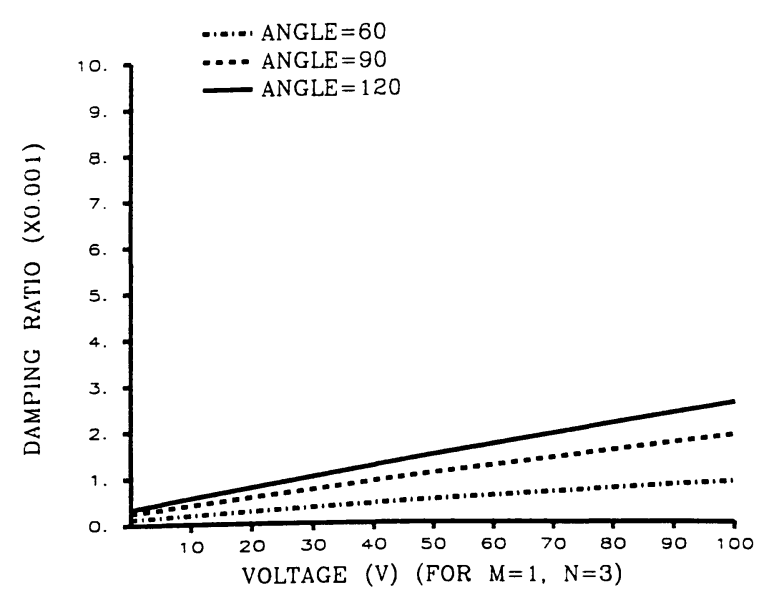

FIGURE 12 Damping control of the piezoelectric shell, $(1,3)$ mode.

control effect to the $(3,1)$ mode was limited. The other two modes, $(m=1, n=1)$ and $(m=1$, $n=3$ ) (Fig. 10, 12), had relatively significant circumferential oscillations. Accordingly, circumferential control effects to these two modes were relatively significant.

Note that the modal damping ratio is inversely proportional to the modal frequency as indicated in the derived damping equation. For the first two modes, $(m=1, n=1)$ and $(m=3, n=1)$, the natural frequencies for the $\beta=120^{\circ}$ shell were higher than those of the $\beta=90^{\circ}$ and $\beta=60^{\circ}$ shells (Tzou, 1993). Thus, the damping ratios were below the other two in Fig. 10 and 11. However, this is reversed for the ( $m=1, n=3)$ mode, as shown in Fig. 12.

\section{SUMMARY AND CONCLUSIONS}

In this article spatially distributed active vibration controls of a laminated cylindrical shell composite and a piezoelectric cylindrical shell were studied, and their spatial filtering characteristics investigated. It was observed that there were inplane membrane control forces and out of plane counteracting control moments in the laminated shell case, and there was only an in-plane circumferential control force in the piezoelectric shell case. Accordingly, the laminated shell was controlled by both membrane control forces and control moments, and the piezoelectric shell was only influenced by the circumferential force. In both cases, the control action to a symmetrical distrib- uted and simply supported boundary condition was limited to odd natural modes and ineffective to even natural modes, due to symmetries of natural modes and also control signal cancellations. Segmenting an actuator into patches can improve the even mode controllability (Tzou and Fu, 1993). Furthermore, only the velocity feedback was considered in both case studies.

Distributed filtering characteristics of the fully distributed actuator laminated on the elastic cylindrical shell were studied. Control actions induced by the fully distributed actuator were primarily for odd natural modes, and ineffective for even modes. Analyses suggested that the in-plane control forces are essential for controlling low natural modes, and this control effect decreases as the mode number increases. The control moment effects basically remained at the same level for all modes calculated. The controlled damping ratio increased linearly with the increase of feedback gains; it decreased as the mode number increased. Note that the overall convergence of a modal time history was determined by the combined effect of the mode natural frequency and the damping ratio. It was also found that the membrane control action remained identical, and the bending control action increased linearly when the elastic shell became thicker. However, the overall damping control decreased because the flexural rigidity was a cubic function of the shell thickness and the control moment was only a linear function.

Damping control of the piezoelectric cylindrical shell was also investigated. A distributed electric in-plane circumferential force, in the $\theta$ direction, was kept in the piezoelastic equation and it was used as a control force in distributed shell controls. Simulation results suggested, in general, that damping ratio was enhanced when the feedback voltage increased. Analytical solutions also showed that this control action is only effective to odd natural modes and ineffective to even modes. Because this control force was primarily in the $\theta$ direction, control effects to circumferential oscillation modes were significant.

It should be noted that in practical applications, the maximum control voltage was limited by a breakdown voltage of piezoelectric materials. The actuation was rather limited, i.e., in submicron strains, for current materials commercially available. In addition, a high feedback voltage could also heat up the piezoelectric actuator or shell. This temperature effect was not considered in this study. 
This research was supported, in part, by a grant from the National Science Foundation (No. RII-8610671) and the Commonwealth of Kentucky. A grant from the Army Research Office (DAAL03-91-G-0065; technical monitor Dr. Gray L. Anderson) is also gratefully acknowledged. Contents of the information do not necessarily reflect the position or the policy of the government, and no official endorsement should be inferred.

\section{REFERENCES}

Baz, A., and Poh, S., 1988, "Performance of an Active Control System with Piezoelectric Actuators, ' Journal of Sound and Vibration, Vol. 126, pp. 327-343.

Birman, V., 1992, Theory of Geometrically Nonlinear Composite Plates with Piezoelectric Stiffeners, DSC-Vol.38, Active Control of Noise and Vibration, 1992 ASME WAM, Anaheim, CA, Nov. 8-13, 1992, pp. 231-237.

Colins, S. A., Miller, D. W., and von Flotow, A. H., 1991, "Piezoelectric Spatial Filters for Active Vibration Control," Recent Advances in Structural Control and Vibration, pp. 65-73.

Hubbard, J. E., and Burke, S. E., "Distributed Transducer Design for Intelligent Structural Components," in H. S. Tzou, and G. L. Anderson, Intelligent Structural Systems, 1992, Kluwer Academic Publishers, Dordrecht/Boston/London, pp. 35-324.

Lee, C. K., and Moon, F. C., 1990, "Modal Sensors/
Actuators," ASME Journal of Applied Mechanics, Vol. 57, pp. 434-441.

Tzou, H. S., 1991, "Distributed Modal Identification and Vibration Control of Continua," ASME Journal of Dynamic Systems, Measurements, and Control, Vol. 113, pp. 494-499.

Tzou, H. S., 1993, Piezoelectric Shells (Distributed Sensing and Control of Continua), Kluwer Academic Publishers.

Tzou, H. S., and Anderson, G. L. (Eds.), 1992, Intelligent Structural Systems, Kluwer Academic Publishers, Dordrecht/Boston/London.

Tzou, H. S., and Fu, H., 1993, "A Study on Segmentation of Distributed Sensors and Actuators, Part-1, Theoretical Analysis; Part-2, Parametric Study and Vibration Controls," Journal of Sound and Vibration, Vol. 172, pp. 247-275.

Tzou, H. S. and Fukuda, T. (Eds.), 1992. Precision Sensors, Actuators, and Systems, Kluwer Academic Publishers, Dordrecht, Boston, London.

Tzou, H. S., and Zhong, J. P., 1993, "Electromechanics and Vibration of Piezoelectric Distributed Systems," ASME Journal of Dynamic Systems, Measurements, and Control, Vol. 115, pp. 506-517.

Tzou, H. S., Zhong, J. P., and Natori, M. C., 1993, "Sensor Mechanics of Distributed Shell Convolving Sensors Applied to Flexible Rings," ASME Journal of Vibration and Acoustics, Vol. 115, pp. 40-46.

Zhong, J. P., 1991, A Study on Piezoelectric Shell Dynamics Applied to Structural Identification and Control, Ph.D. Thesis, Department of Mechanical Engineering, University of Kentucky. 

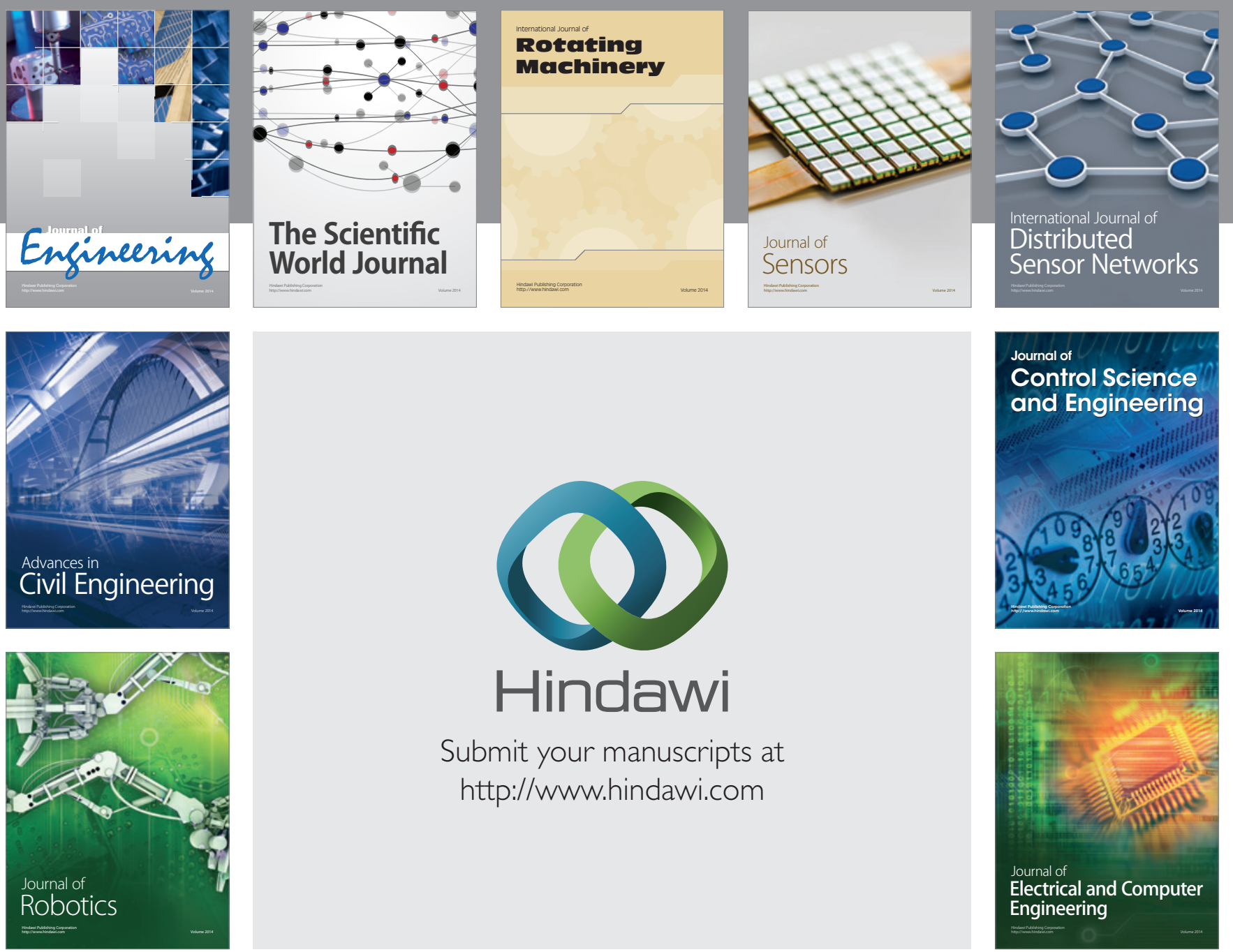

Submit your manuscripts at

http://www.hindawi.com
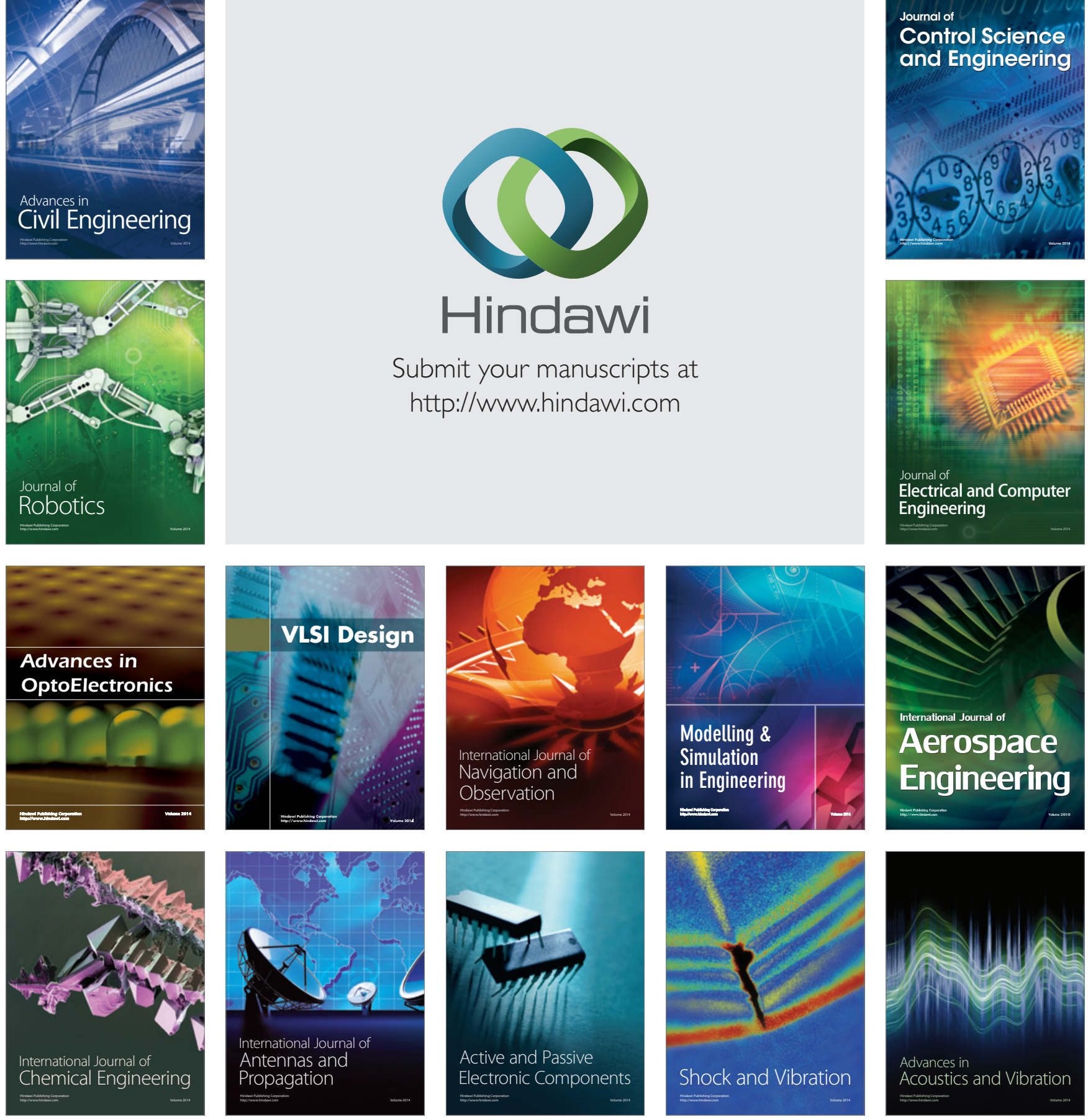